\title{
Fonctionnalisation de surfaces d'acier inoxydable par des enzymes en vue d'inhiber l'adhésion de bactéries et la formation de biofilms en eau de mer
}

\author{
A. Caro ${ }^{1}$, V. Humblot ${ }^{1}$, M. Minier ${ }^{2}$, M. Salmain ${ }^{2}$, C. Compère ${ }^{3}$ \\ et C.-M. Pradier ${ }^{1}$ \\ 1 Laboratoire de Réactivité de Surface, UMR CNRS 7609, UPMC - Paris 6, Tour 54/55, Case 178, 4 place \\ Jussieu, 75252 Paris Cedex 05, France \\ e-mail : caro@ccr.jussieu.fr; humblot@ccr.jussieu.fr; Claire-Marie.Pradier@ccr.jussieu.fr \\ 2 Laboratoire de Chimie et Biochimie des Complexes Moléculaires, UMR CNRS 7576, ENSCP, 11 rue \\ Pierre et Marie Curie, 75231 Paris Cedex 05, France \\ e-mail : michel-minier@enscp.fr; michele-salmain@enscp.fr \\ 3 Service ERT/IC, IFREMER Centre de Brest, BP. 70, 29280 Plouzané, France \\ e-mail : chantal.compere@ifremer.fr
}

Mots-clés :

Acier inoxydable; fonctionnalisation; enzymes; adsorption bactérienne; PM-IRRAS; XPS

\section{Key words:}

Stainless steel; functionalization; enzymes; bacterial adsorption; IR; XPS

\begin{abstract}
Résumé - Tout matériau immergé en milieu marin se recouvre de composés azotés, protéines, carbohydrates, sels, silice, puis de bactérie en quelques heures, et d'un biofilm mature en quelques jours. Cela altère ses propriétés physico-chimiques, entraîne un risque accru de corrosion localisée et de biodétérioration, avec pour conséquences des coûts d'entretien et de nettoyage importants. Les peintures contenant des sels de tributylétain (TBT) utilisées jusqu'à présent pour protéger ces surfaces se sont avérées toxiques pour le milieu marin [1]. Elles sont désormais soumises à des normes internationales, avec une interdiction d'application des sels TBT effective depuis 2003, et une interdiction totale en 2008. II est donc aujourd'hui nécessaire de rechercher des alternatives à ces peintures. La solution ici proposée consiste à agir sur les premières étapes de formation du biofilm, à savoir la fixation de protéines et des premières bactéries sur la surface. Pour cela, la surface est modifiée chimiquement et fonctionnalisée par des enzymes, lesquelles vont agir localement sur la bactérie par hydrolyse de certains constituants de sa paroi, empêchant ainsi son adhésion à la surface. Les différentes étapes du greffage ont été contrôlées par Infra Rouge et par Spectroscopie de Photoélectrons X. Ces analyses ont permis de valider chaque étape du greffage. En parallèle, un nouveau test enzymatique pour tester l'activité de l'enzyme greffée a été mis en place et validé. II a permis de vérifier l'efficacité de l'enzyme greffée vis-à-vis d'une suspension bactérienne modèle.
\end{abstract}

\begin{abstract}
Surface Modification of stainless steel by grafting enzymes to prevent bacterial adhesion and biofilms development in seawater. All surfaces that are immersed in seawater rapidly become covered by nitrogenous compounds, proteins, carbohydrates, salts, silica and bacteria in a few hours, and by a grown biofilm in a few days. These biodeterioration, physical and chemical damages involve important cleaning costs. Organostanic paintings used up to now to protect the surfaces against this spoiling will be prohibited in 2008 because of their toxicity. That's why the stake today is to find alternatives to these paintings. The solution which is here suggested consists in acting on the first stages of the formation of the biofilm i.e. the proteins and bacterial adsorption on the surface. For this, the surface is chemically modified then functionalized by enzymes. Enzymes will act locally on the bacteria and thus prevent their adsorption on the surface. The different stages of the grafting are controlled by Infra Red and X-Ray Photoelectron Spectroscopy. In addition, a new enzymatic test making it possible to test the activity of the grafted enzyme was set up and validated. It enables to check the effectiveness of the grafted enzyme towards a model bacterial suspension.
\end{abstract}

es recherches dans le domaine des surfaces à propriétés « antifouling » s'articulent essentiellement aujourd'hui autour de deux axes :

1-modifier la formulation des peintures et leur mode d'action [2-5], 2-trouver des alternatives telles que l'approche biomimétique [6], la modification chimique des surfaces par des polymères [7-10], ou par des enzymes [11-13].

Ce travail s'intéresse à la modification chimique des surfaces qui consiste à immobiliser sur celles-ci des molécules à propriétés anti-adhésives ou 
biologiques qui vont agir sur les premières étapes de la formation du biofilm à savoir l'adsorption de protéines puis l'adhésion de bactéries marines sur le matériau.

Parmi les stratégies possibles : 1'immobilisation de Poly (éthylène glycol) (PEG) est sans doute la plus étudiée à ce jour. Ces polymères présentent de nombreux atouts. Dans un premier temps, ils possèdent des caractéristiques physico-chimiques intéressantes telles que leur biocompatibilité, leur solubilité dans l'eau et dans de nombreux solvants organiques, leur large gamme de poids moléculaires et de fonctionnalités, ce qui laisse envisager de nombreuses voies d'utilisation. Mais surtout, en solution, les chaînes polymères du PEG s'hydratent fortement et créent ainsi une barrière stérique autour d'elles empêchant les interactions non spécifiques surface-protéines [10,14-20].

Cependant leur efficacité à long terme contre la formation de biofilm en milieu marin reste à prouver [21].

Dans cette étude, nous avons choisi une autre voie de greffage : la voie enzymatique qui consiste à greffer une enzyme sur la surface de manière à lui conférer des propriétés biologiques capables de limiter l'adhésion des bactéries environnantes.

La surface métallique étudiée est l'acier inoxydable, impliqué comme matériau de structure dans de nombreuses installations marines.

Plusieurs études de fonctionnalisation de l'acier reposent sur la silanisation de la surface $[22,23]$. Or les silanes polymérisés sont instables en milieu aqueux. Une autre voie possible est la modification de la surface via un polymère polycationique qui va permettre d'introduire au niveau de la surface une forte densité de groupements fonctionnels [24]. C'est cette voie qui a été suivie dans cette étude. Le greffage comporte trois étapes :

1. fixation du polymère cationique : le Polyéthylènimine (PEI), sur la surface métallique,

2. fixation d'un bras espaceur, le Glutaraldéhyde (GA), qui va jouer deux rôles : l'introduction d'une fonction aldéhyde qui réagira ultérieurement avec l'enzyme et l'ajout d'un espace entre la surface et l'enzyme qui permettra de protéger les fonctions biologiques de l'enzyme,

3. fixation de l'enzyme à propriété antibactérienne. L'enzyme utilisée est le lysozyme HEWL (Hen Egg-White
Lysozyme). Cette enzyme hydrolyse les glucides constitutifs des parois bactériennes, et provoque ainsi la lyse de ces bactéries [25].

Les différentes étapes de greffage seront caractérisées par PM-IRRAS (Polarization Modulation Infrared Reflection Absorption Spectroscopy) pour la caractérisation des fonctions chimiques présentes à la surface du matériau et XPS (X-Ray Photoelectron Spectroscopy) pour l'analyse chimique élémentaire du matériau en surface et de la couche adsorbée. L'activité biologique de l'enzyme sera ensuite testée vis-à-vis d'une suspension bactérienne selon un protocole mis au point au laboratoire.

\section{Matériels et méthodes}

\section{Matériels}

Polyéthylènimine (branché, $M_{\mathrm{w}} \sim 25000$ ), Glutaraldéhyde (GA) $25 \%$ dans l'eau (w/v), et Lysozyme (Hen egg white lysozyme) (HEWL) (50,000 unités/mg protéine), cristallisés trois fois, ont été obtenus chez SigmaAldrich. La souche bactérienne Micrococcus Luteus a été fournie par la Collection Institut Pasteur. Les coupons d'acier inoxydable AISI $316 \mathrm{~L}(10 \times 10 \times 2 \mathrm{~mm})$, polis sur une face à $0,5 \mu \mathrm{m}$, ont été fournis par Goodfellow. L'acide sulfurique $96 \%$ a été obtenu chez Carlo Erba Reactifs et le bichromate de potassium chez Prolabo. L'acide sulfochromique a été préparé par dissolution de $6 \mathrm{~g}$ de bichromate de potassium dans $100 \mathrm{~mL}$ d'acide sulfurique, à $60{ }^{\circ} \mathrm{C}$ pendant 10 minutes.

\section{Méthodes}

\section{Nettoyage des coupons}

Après avoir été polis, les coupons sont rincés à l'éthanol (20 min) puis lavés dans des bains ultrasons, successivement dans le cyclohexane (15 min), l'eau (3×10 min) et l'acétone (20 $\mathrm{min})$.

\section{Prétraitement des coupons}

Les coupons sont traités à l'acide sulfochromique à $60{ }^{\circ} \mathrm{C}$ pendant $10 \mathrm{~min}$, puis rincés intensément à l'eau $(3 \times 30 \mathrm{~min})$ et séchés sous courant d'azote. Les surfaces ainsi traitées sont désignées par l'abréviation ss-sc. 


\section{Greffage du Polyéthylènimine (PEI)}

Les surfaces ss-sc sont immergées dans une solution de PEI (3\% (w/v) dans l'eau) pendant $3 \mathrm{~h}$ sous agitation magnétique. Elles sont ensuite rincées dans l'eau $(3 \times 15 \mathrm{~min})$ et séchées sous flux d'azote. Ces coupons seront nommés ss-sc-PEI ou PEI.

\section{Immobilisation de I'enzyme HEWL}

Les coupons ss-sc-PEI sont immergés une nuit dans la solution de glutaraldéhyde (GA) (solution à $5 \%$ dans l'Éthanol absolu), puis rincés successivement dans l'éthanol (15 min) et dans l'eau (15 min), avant d'être séchés sous courant d'azote. Ces coupons, appelés ss-sc-PEI-GA ou GA, sont ensuite immergés une nuit, à $4{ }^{\circ} \mathrm{C}$, dans une solution d'enzyme ( $1 \mathrm{mg} / \mathrm{mL}$ dans le tampon PBS pH $8,0)$. Ils sont rincés dans cette même solution tampon PBS pH 8,0 $(3 \times 20 \mathrm{~min})$ puis séchés sous azote. Ces surfaces sont désignées par l'abréviation ss-sc-PEI-GA-HEWL ou HEWL.

L'ensemble de la fonctionnalisation est schématisé sur la figure 1.

\section{Analyses PM-IRRAS}

Les données PM-IRRAS sont enregistrées sur un spectromètre Nicolet (FT-IR 5700) équipé d'un détecteur MCT. L'angle d'incidence est de $85^{\circ}$. Les acquisitions sont effectuées après chaque étape du greffage. Les spectres sont collectés sur 128 scans avec une résolution spectrale de $8 \mathrm{~cm}^{-1}$.

\section{Analyses XPS}

L'analyseur XPS utilisé est un analyser PHOIBOS 100 de chez SPECS, avec comme source de rayons X la raie $\mathrm{Mg} \mathrm{K} \alpha$ à 1253,6 eV. Les spectres ont été réalisés avec une énergie de passage de 10 eV. L'énergie de liaison des éléments a été calibrée sur l'énergie de liaison du chrome Cr2p3/2 du chrome oxydé. L'intensité des pics des différents éléments analysés a été corrigée par le facteur de Scofield [26].

\section{Efficacité de l'enzyme greffée}

En parallèle des analyses physico-chimiques de surface, un nouveau test enzymatique permettant de tester l'efficacité de l'enzyme

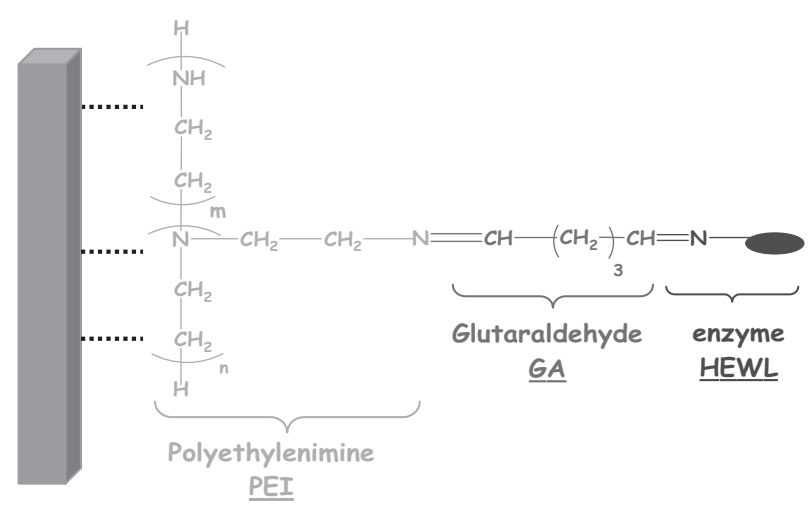

Fig. 1. Fonctionnalisation de la surface d'acier inoxydable : SS-SC-PEI-GA-HEWL.

Fig. 1. Stainless steel surface functionalization: SS-SC-PEIGA-HEWL.

greffée a été mis au point et validé. Il a permis de vérifier l'efficacité de la modification de la surface d'acier vis-à-vis d'une suspension bactérienne modèle.

La souche bactérienne utilisée est la souche Micrococcus luteus ATCC 4698 (10 ${ }^{9}$ à $10^{10}$ bactéries $\left./ \mathrm{mL}\right)$, cultivée dans un milieu nutritif Trypto-Caseine-Soja (3\% (w/v) dans l'eau). Une goutte de $50 \mu \mathrm{L}$ de suspension bactérienne est déposée sur le coupon à étudier avec une micropipette, la goutte est laissée à incuber une nuit sous atmosphère humide (saturée), avant d'être récupérée avec la micropipette et étalée sur boîte de Pétri (milieu Trypto-Caseine-Soja Agar). Après quelques jours (3 à 5 jours), les colonies de bactéries qui se sont développées sont comptées.

Trois sortes d'échantillons sont étudiées : goutte déposée sur un coupon non fonctionnalisé, goutte déposée sur un coupon fonctionnalisé, et goutte de référence directement prélevée de la suspension bactérienne (Fig. 7).

Pour vérifier que l'enzyme reste fixée au support et n'est pas libérée dans la goutte de suspension bactérienne déposée sur la surface, un test dit de désorption a été mis au point. Il consiste à déposer une goutte de tampon PBS, exempte de toute bactérie, sur les surfaces à étudier, dans les mêmes conditions que précédemment, à récupérer cette goutte de tampon et à la mélanger au même volume de suspension bactérienne. Ce mélange est laissé à incuber une nuit puis est étalé sur milieu Agar. L'échantillonnage est le même que précédemment. Si aucune diminution du nombre de colonies n'est observée pour la goutte déposée sur la surface fonctionnalisée par rapport à une goutte 


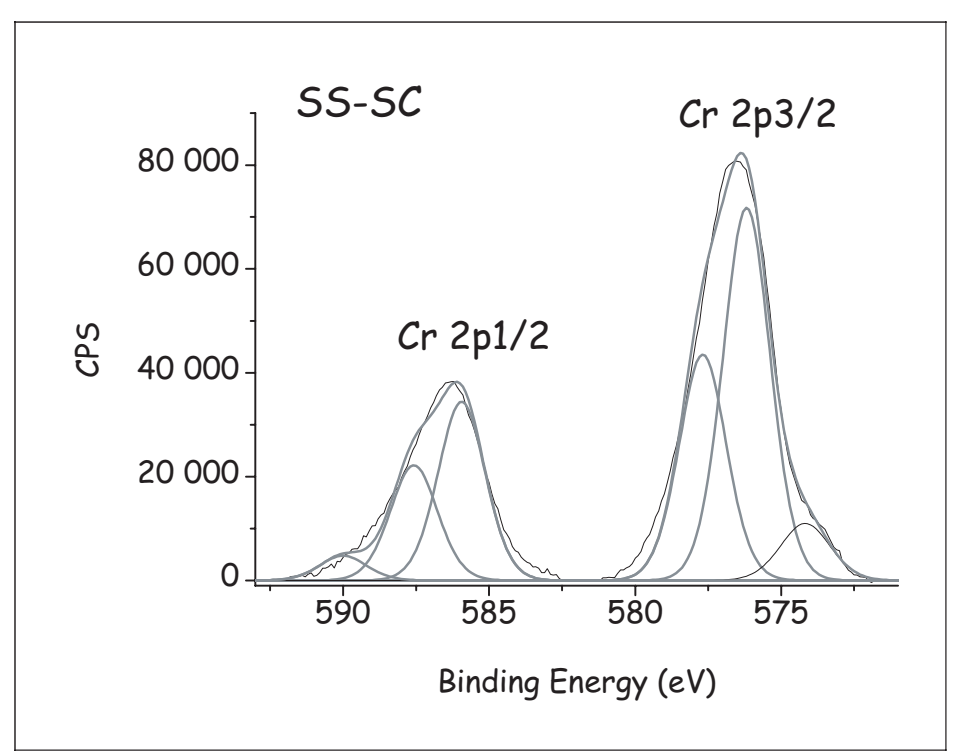

Fig. 2. Région du pic XPS Cr2p de l'échantillon ss-sc.

Fig. 2. High-resolution $\mathrm{Cr} 2 p$ spectra of stainless ss-sc.

déposée sur surface vierge, c'est que l'enzyme n'est pas libérée en quantité mesurable (Fig. 9).

\section{Resultats et discussion}

\section{Caractérisation par XPS des échantillons après les différentes étapes du greffage}

Les surfaces d'acier inoxydable sont préalablement traitées par l'acide sulfochromique de manière d'une part, à supprimer les impuretés présentes en surface, et d'autre part, à créer une couche oxydée hydroxylée réactive pour la suite du traitement. Après immersion dans la solution d'acide sulfochromique, les échantillons sont rincés plusieurs fois à l'eau $(3 \times 20 \mathrm{~min})$ et analysés par IR et XPS.

L'analyse XPS de l'échantillon ss-sc apporte des informations sur les éléments présents en surface à savoir principalement les éléments : $\mathrm{Cr}, \mathrm{Fe}, \mathrm{Ni}, \mathrm{O}$ et $\mathrm{C}$ et les liaisons chimiques dans lesquelles chacun est impliqué. Le signal du $\mathrm{Cr}$ peut être décomposé en 3 contributions à 574,3 ; 576,2 ; et $577,7 \mathrm{eV}$ attribuées respectivement au $\mathrm{Cr}$ métallique, à l'oxyde de chrome $\left(\mathrm{Cr}_{2} \mathrm{O}_{3}\right)$ et à l'hydroxyde de chrome $\left(\mathrm{Cr}(\mathrm{OH})_{3}\right)$, les oxydes et hydroxydes de chrome étant largement majoritaires ( $90 \%$ du signal total du chrome $\mathrm{Cr} 2 \mathrm{p} 3 / 2$ ) par rapport au chrome métal (10\% du signal). L'analyse XPS permet ainsi de vérifier la présence d'une couche d'oxydes et d'hydroxydes de chrome à la surface de l'échantillon sulfochromé (Fig. 2).
Tableau 1. Contributions des pics XPS O1s après chaque étape du greffage et pourcentage de leurs aires.

Table 1. Deconvolution of high-resolution O1s peak and measurement of $\%$ areas.

\begin{tabular}{|l|c|c|c|}
\hline & $\begin{array}{c}\mathrm{Cr}_{2} \mathbf{O}_{3} \\
\mathbf{5 3 0} \mathbf{~ e V}\end{array}$ & $\begin{array}{c}\mathrm{Cr}(\mathbf{O H})_{3} \mathbf{~ o u} \\
\mathbf{R}-\mathbf{O H} \\
\mathbf{5 3 1 , 5} \mathbf{~ e V}\end{array}$ & $\begin{array}{c}\mathbf{C}-\mathbf{O} \\
\mathbf{5 3 2 , 5} \mathbf{~ e V}\end{array}$ \\
\hline ss-Sc & 51,4 & 41 & 7,6 \\
\hline PEI & 38,1 & 46,6 & 15,3 \\
\hline GA & 15,7 & 52,8 & 31,4 \\
\hline HEWL & 10,5 & 66,4 & 23,1 \\
\hline
\end{tabular}

Les figures 3 et 4 montrent les spectres $\mathrm{O} 1 \mathrm{~s}$ et $\mathrm{C} 1 \mathrm{~s}$ respectivement après chaque étape de greffage. L'oxygène peut être décomposé en 3 contributions à $530 ; 531,5$ et $532,5 \mathrm{eV}$ respectivement attribuées aux oxygènes de l'oxyde de chrome, aux oxygènes de l'hydroxyde de chrome, et aux oxygènes de la liaison simple $\mathrm{C}-\mathrm{O}$ présente dans un composé organique (Fig. 3). Après traitement à l'acide sulfochromique, les oxygènes présents en surface proviennent principalement des oxydes et hydroxydes de chrome (plus de $90 \%$ de l'intensité) (Tab. 1).

Si le chrome et l'oxygène sont les éléments majoritaires présents à la surface de l'échantillon ss-sc, respectivement 42 et $40 \%$ des éléments présents (Fig. 5), du carbone est également détecté (environ $4 \%$ ). Le signal du carbone se décompose en 3 contributions à 285 (liaisons C-C et C-H), 286,6 (liaisons CO) et 288,6 eV (liaisons C=O) (Fig. 4). Le carbone détecté provient ainsi essentiellement d'une contamination de la surface par des hydrocarbures et des carbonates.

Après immersion dans la solution de PEI et rinçage, les coupons sont analysés par XPS (cf. spectres O1s et C1s Figs. 3 et 4). Le signal de l'azote à $400 \mathrm{eV}$, absent de l'échantillon avant traitement au PEI, est présent à hauteur de $11 \%$ après l'étape de greffage (Fig. 5). Parallèlement, l'atténuation des signaux relatifs au chrome et à l'oxygène confirme le recouvrement de la surface métallique oxydée par le PEI. L'épaisseur moyenne de la couche greffée est évaluée à 2,6 nm, cette épaisseur étant calculée à partir de la formule suivante:

$$
I / I_{0}=\exp (-d / \lambda \sin \theta)
$$

avec $I / I_{0}$ le rapport entre les intensités $I_{0}$ du Cr (ss-sc) et I du Cr (PEI), $d$ l'épaisseur moyenne du film greffé, $\theta$ l'angle entre l'analyseur et la surface $\left(90^{\circ}\right)$ et $\lambda$ la longueur d'atténuation des photoélectrons du Cr2p à travers une couche organique $(2,9 \mathrm{~nm})$ [27]. 

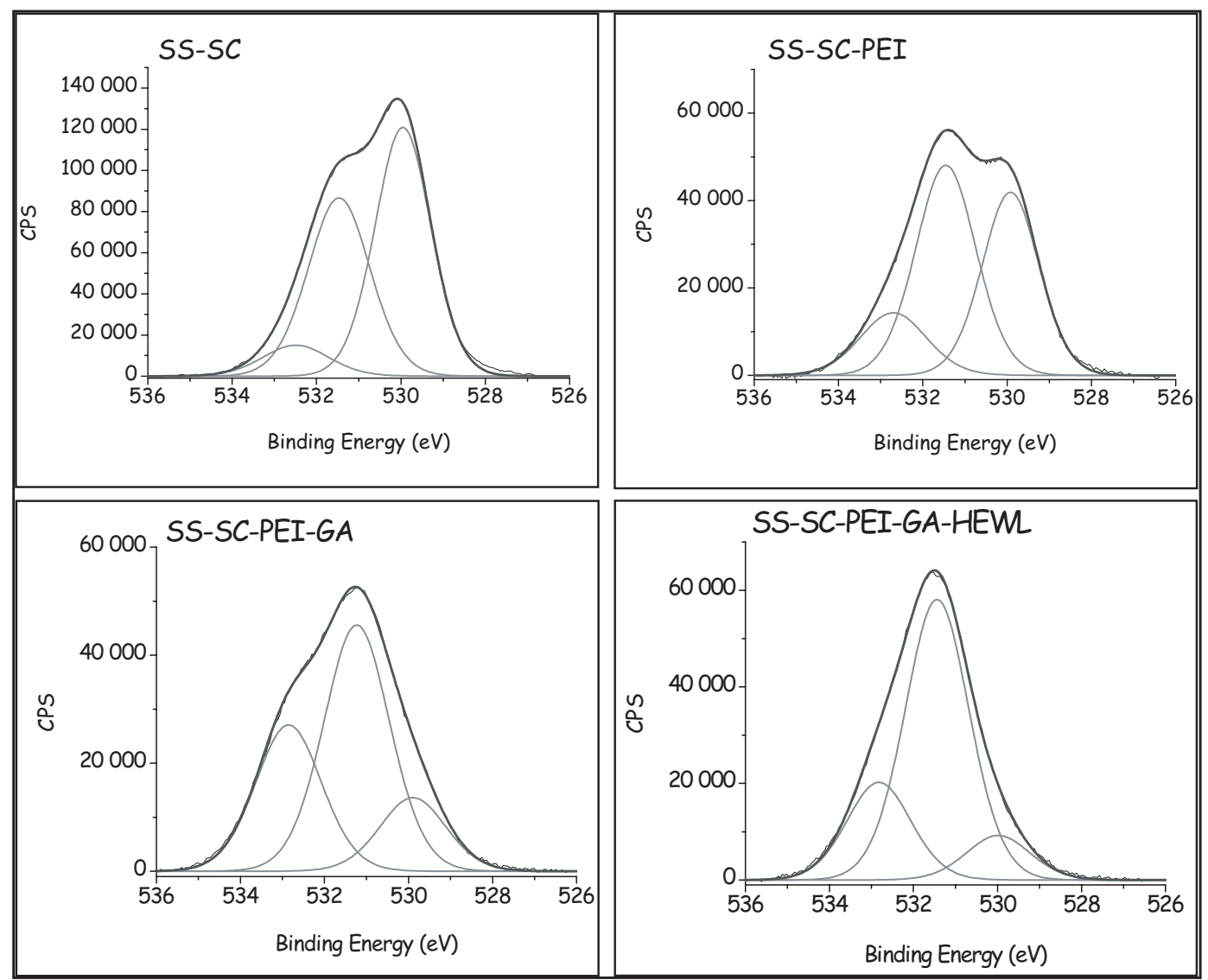

Fig. 3. Pic XPS de l'oxygène O1s après chaque étape du greffage.

Fig. 3. High-resolution O1s spectra of stainless after successive chemical treatments.

Cependant, les signaux relatifs aux métaux sont encore détectés (Fig. 5), ce qui s'explique par la faible épaisseur du PEI. Le signal du carbone peut être décomposé en 2 pics principaux à 286,1 et $288,4 \mathrm{eV}$ (Fig. 4), relatifs respectivement aux liaisons $\mathrm{C}-\mathrm{N}$, et aux $\mathrm{C}=\mathrm{O}$ de surface. De plus le rapport $\mathrm{N} / \mathrm{C}$ (contribution à $286.1 \mathrm{eV})=0,55$ est très proche du rapport théorique $\mathrm{N} / \mathrm{C}=0,5$ de la molécule de PEI.

L'analyse XPS permet de confirmer le recouvrement de la surface par le GA avec l'atténuation du signal du chrome au profit des éléments carbone et oxygène présents dans le GA (Fig. 5). L'étude du signal du carbone (Fig. 4 et Tab. 2) fait apparaître 4 contributions à 285,4 (C-C, C-H), 286,4 (C-N), 288.3 $(\mathrm{C}=\mathrm{O})$ et $289,3 \mathrm{eV}$ (carbonates). De plus, l'intensité du signal de l'azote reste stable (Fig. 5) ce qui est cohérent avec le recouvrement de la couche de PEI par le GA qui ne contient pas d'azote.

Après immersion dans la solution d'enzymes et plusieurs rinçages, l'étude XPS du signal relatif au carbone (Fig. 4 et Tab. 2) révèle la présence de l'enzyme avec une contribution sous forme d'épaulement à
288,1 eV, caractéristique des liaisons peptidiques. Deux autres contributions sont attribuées aux liaisons C-C, C-H $(285,1 \mathrm{eV})$ et C-O $(286,4 \mathrm{eV})$. L'étude de l'oxygène montre une augmentation de la contribution à $531,4 \mathrm{eV}$ (Fig. 3 et Tab. 1) qui peut être attribuée aux fonctions $\mathrm{OH}$ présentes dans l'enzyme. L'étude de l'évolution de la proportion des différents éléments montre une augmentation des 3 éléments $C, O$ et $N$ et une atténuation du signal du chrome. L'épaisseur moyenne de la couche greffée est évaluée à $3,8 \mathrm{~nm}$ ce qui correspond à environ une monocouche d'enzymes greffées.

\section{Caractérisation par PM-IRRAS des échantillons après les différentes étapes du greffage}

En IR, aucune fonction chimique n'est détectée à la surface du coupon d'acier inoxydable ss-sc traité par l'acide sulfochromique : intensités des signaux inférieures au seuil de détection de la technique. Après traitement du coupon par une solution de PEI, la caractérisation IR permet d'identifier les fonctions chimiques caractéristiques du 

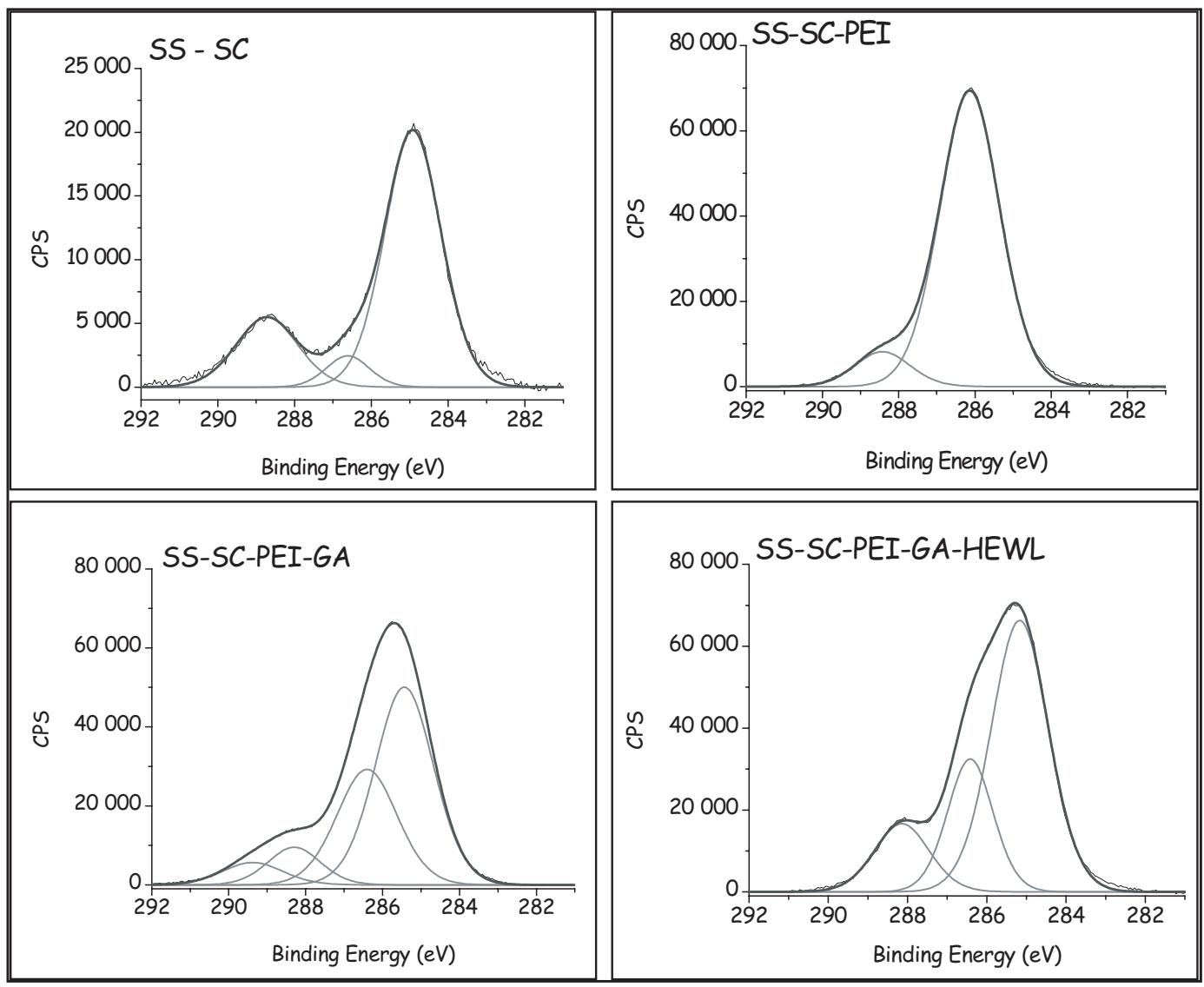

Fig. 4. Pic XPS du carbone C1s après chaque étape du greffage.

Fig. 4. High-resolution C1s spectra of stainless after successive chemical treatments.

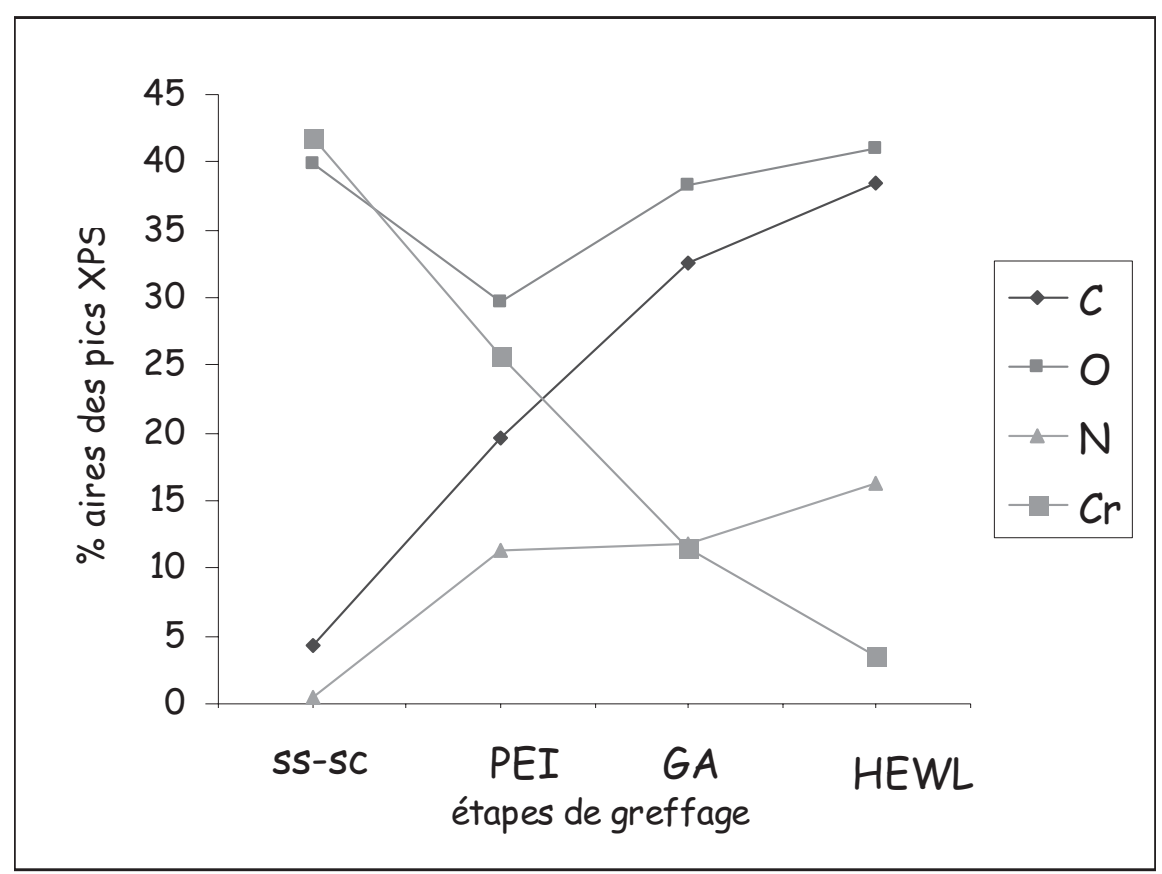

Fig. 5. Évolution du pourcentage relatif à chaque élément au cours des différentes étapes du greffage.

Fig. 5. C, O, N and Cr percentage after successive chemical treatments. 
Tableau 2. Contributions des pics XPS C1s après chaque étape de greffage et pourcentage de leurs aires.

Table 2. Deconvolution of high-resolution C1s peak and measurement of $\%$ areas.

\begin{tabular}{|l|c|c|c|c|c|}
\hline & $\begin{array}{c}\text { C-C } \\
\text { C-H }\end{array}$ & $\begin{array}{c}\text { C-O } \\
\text { C-N }\end{array}$ & $\begin{array}{c}\text { C=O } \\
\text { C=N }\end{array}$ & $\begin{array}{c}\text { Peptides } \\
\text { O-C=O } \\
\text { O-C=N } \\
\mathbf{2 8 8 , 1 ~ e V ~}\end{array}$ & carbonates \\
\hline ss-sc & 71,8 & $6, \mathbf{2 8} \pm \mathbf{0 , 4} \mathbf{~ e V}$ \\
\hline PEI & & 90,2 & & & 21,5 \\
\hline GA & 52,8 & 31,8 & 9,3 & & 9,8 \\
\hline HEWL & 61,4 & 23,9 & \multicolumn{2}{|c|}{14,7} & 6,2 \\
\hline
\end{tabular}

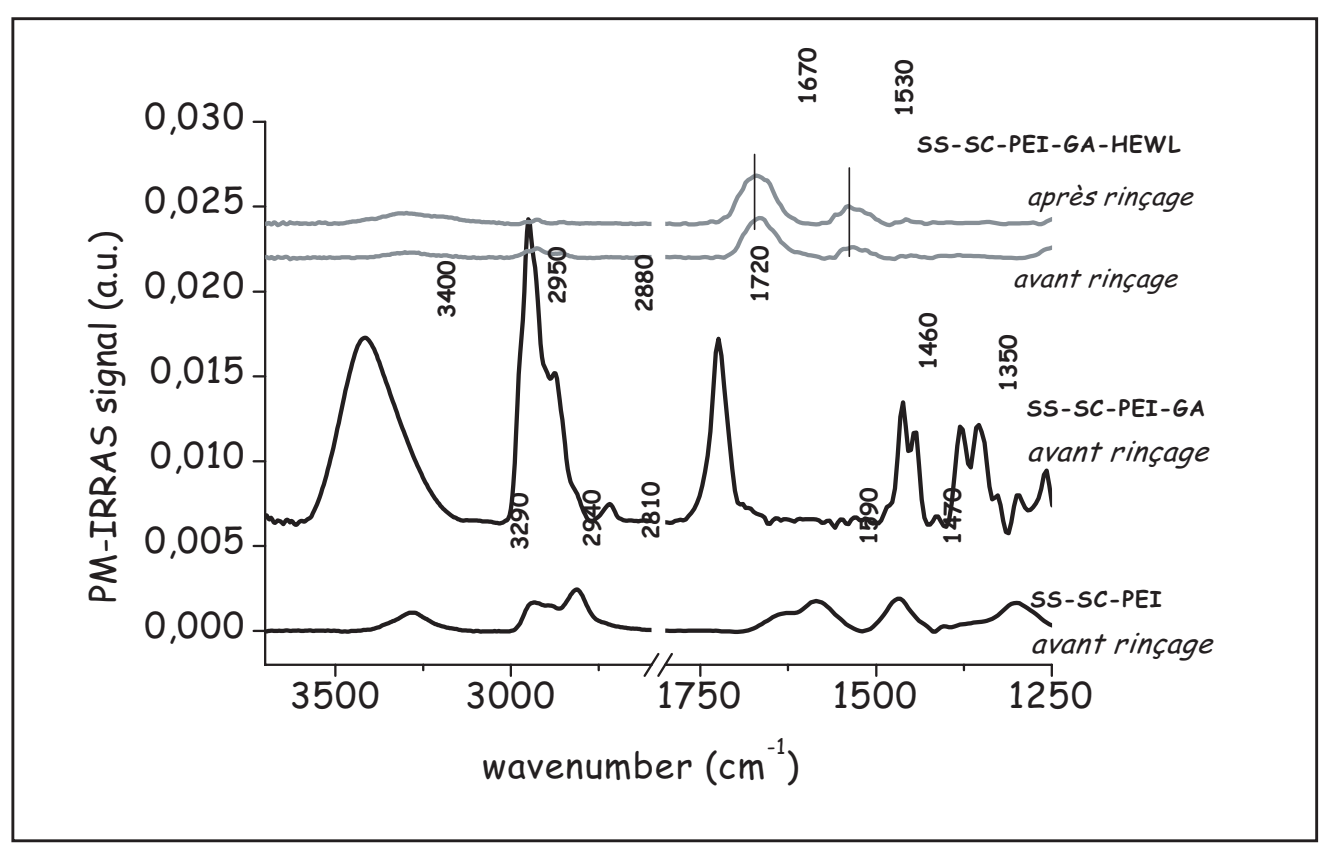

Fig. 6. Caractérisation par PM-IRRAS des différentes étapes de greffage : 1-PEI ; 2-GA ; 3-Enzyme. Fig. 6. PM-IRRAS spectra of 1-SS-SC-PEI, 2-SS-SC-PEI-GA and 3-SS-SC-PEI-GA-HEWL.

polymère : liaisons $\mathrm{C}-\mathrm{H}$ (vers $2900 \mathrm{~cm}^{-1}$ et $1450 \mathrm{~cm}^{-1}$ ) et fonctions amines à $1580 \mathrm{~cm}^{-1}$ (Fig. 6 et Tab. 3). Mais après plusieurs rinçages à l'eau, ces bandes IR diminuent fortement. Après immersion dans la solution de GA, les fonctions chimiques caractéristiques de la molécule sont identifiées par IR, en particulier la fonction aldéhyde à $1720 \mathrm{~cm}^{-1}$ (Fig. 6 et Tab. 3). Mais comme pour le PEI, leur intensité diminue fortement après rinçage. Enfin, le traitement de la surface par l'enzyme lysozyme est étudié par IR. L'IR confirme le greffage de l'enzyme sur la surface et sa stabilité en milieu aqueux pendant plusieurs heures. En effet, les deux bandes amides caractéristiques des protéines sont identifiées à 1650 et $1550 \mathrm{~cm}^{-1}$ (Fig. 6 et Tab. 3). Après plusieurs bains de rinçages d'une durée totale de 2 heures, aucune diminution des intensités de ces deux bandes n'est observée, ce qui tend à prouver qu'aucun relargage de l'enzyme en solution n'a lieu. La réalisation de chaque étape du greffage a été validée par IR et XPS. Il faut maintenant étudier l'action de l'enzyme greffée vis-à-vis d'une suspension bactérienne.

\section{Evaluation de l'activité anti-adhésion de l'enzyme immobilisée sur la surface}

Un test de mesure de l'activité enzymatique de surface, basé sur l'inactivation de la croissance bactérienne, a été mis au point au laboratoire. Pour cela, le rapport entre la surface du coupon analysé et le volume de suspension bactérienne déposé a été optimisé. En effet, jusqu'à présent, les tests de mesure de l'activité enzymatique consistaient à analyser, par spectroscopie UV/visible, l'évolution de la turbidité d'une suspension bactérienne inactive (substrat non vivant), dans lequel était immergée la surface fonctionnalisée. Or les réacteurs utilisés pour ce type de mesures présentent un volume assez important 
Tableau 3. Bandes de vibrations IR caractéristiques de chaque étape de greffage. Table 3. Infrared characteristic group vibrations of PEI; GA; and HEWL.

\begin{tabular}{|c|c|c|c|}
\hline $\begin{array}{l}\text { Étape de la } \\
\text { fonctionnalisation }\end{array}$ & $\begin{array}{l}\text { Groupements fonctionnels } \\
\text { caractéristiques }\end{array}$ & $\begin{array}{l}\text { Longueurs } \\
\text { d'onde }\left(\mathrm{cm}^{-1}\right)\end{array}$ & $\begin{array}{l}\text { Intensités } \mathrm{s}=\text { strong; } \\
\mathrm{m}=\text { medium } ; \mathrm{w}=\text { weak }\end{array}$ \\
\hline 1- PEI & $\begin{array}{l}v \mathrm{~N}-\mathrm{H} \\
v \mathrm{C}-\mathrm{H} \\
\delta \mathrm{N}-\mathrm{H} \text { (amine I, II) } \\
\delta \mathrm{C}-\mathrm{H}\end{array}$ & $\begin{array}{c}3400 \\
2950-2850 \\
\approx 1580 \\
\approx 1450\end{array}$ & $\begin{array}{c}\mathrm{m}-\mathrm{s} \\
\mathrm{m}-\mathrm{s} \\
\mathrm{m}-\mathrm{s} \\
\mathrm{s}\end{array}$ \\
\hline 2- GA & $\begin{array}{l}v \mathrm{O}-\mathrm{H} \\
v \mathrm{C}-\mathrm{H} \\
v \mathrm{C}=\mathrm{O} \text { (aldéhyde) } \\
\delta \mathrm{C}-\mathrm{H}\end{array}$ & $\begin{array}{c}3400 \\
2950-2800 \\
1750-1720 \\
\approx 1450\end{array}$ & $\begin{array}{c}\mathrm{s} \\
\mathrm{m}-\mathrm{s} \\
\mathrm{s} \\
\mathrm{s}\end{array}$ \\
\hline 3- HEWL & $\begin{array}{l}v \mathrm{C}=\mathrm{O} \text { (amide } \mathrm{I}) \\
\delta \mathrm{N}-\mathrm{H}+v \mathrm{C}-\mathrm{N} \text { (amide II) }\end{array}$ & $\begin{array}{l}\approx 1670 \\
\approx 1530\end{array}$ & $\begin{array}{l}\mathrm{s} \\
\mathrm{s}\end{array}$ \\
\hline
\end{tabular}

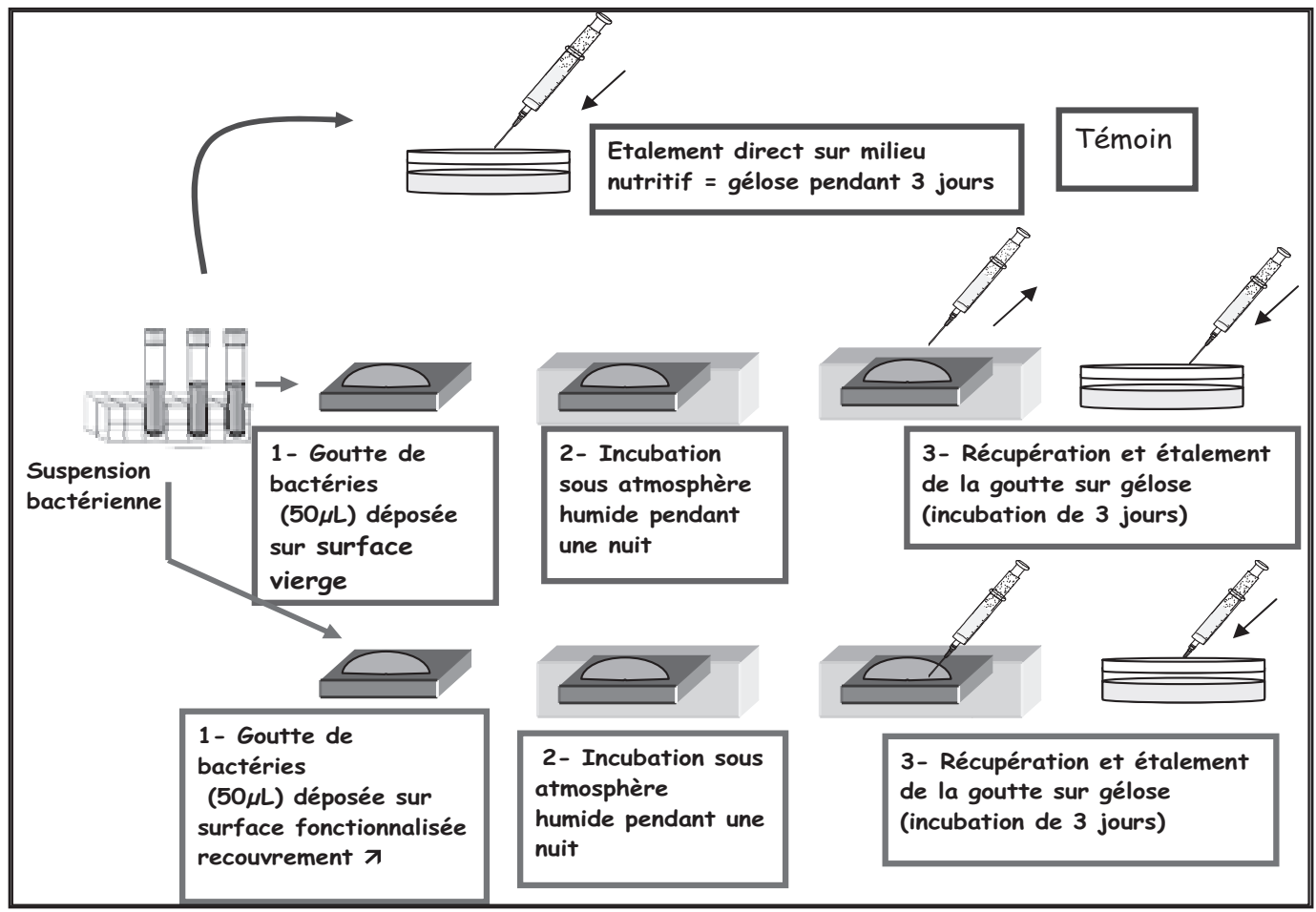

Fig. 7. Principe du test enzymatique local.

Fig. 7. Surface enzymatic test.

(plusieurs $\mathrm{mL}$ ) : le rapport (volume de suspension/aire du coupon) est trop élevé pour avoir une estimation fiable de l'activité de l'enzyme sur les bactéries en contact avec la surface. L'enzyme agissant localement, le domaine de travail doit se limiter au volume local, c'est-à-dire en contact avec la surface. Il a donc fallu imaginer un nouveau test de surface, qui réponde à ces exigences, l'élaborer et le valider. L'idée est de déposer sur le coupon une microgoutte de suspension bactérienne et d'étudier l'action de l'enzyme sur cette microgoutte : le rapport volume de suspension/aire du coupon est alors minimisé et la mesure revient à un contrôle de l'activité enzymatique local vis-à-vis des bactéries en contact direct avec la surface. Le principe de ce test de surface peut être schématisé par la figure 7 .

Le nombre de colonies bactériennes obtenu à partir de la goutte « témoin » (goutte directement prélevée de la suspension bactérienne et étalée sur gélose) et celui obtenu à partir de la goutte « référence » (goutte déposée sur surface nue) sont du même ordre de grandeur à savoir $10^{8} \mathrm{cfu} / \mathrm{mL}$. Pour certains essais, le nombre de colonies bactériennes issu de la référence est inférieur à celui du témoin : cela pouvant être attribué aux conditions opératoires (perte de volume 


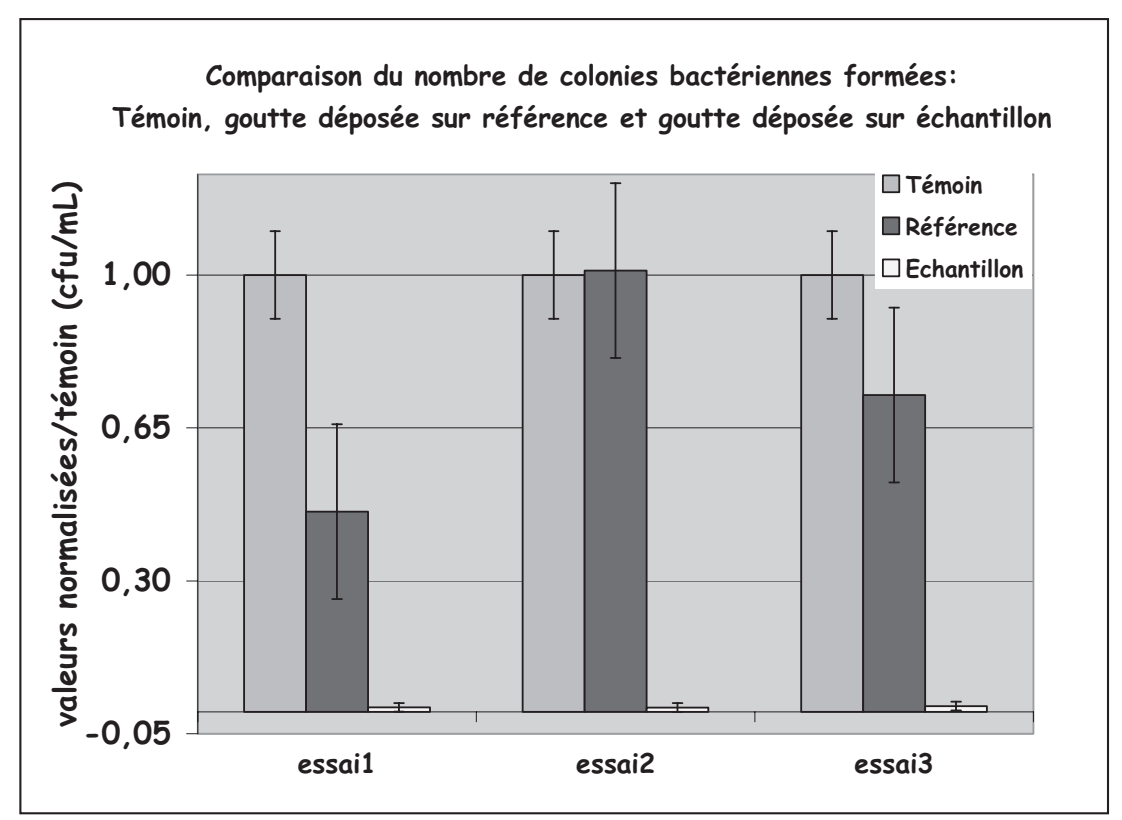

Fig. 8. Résultats du test enzymatique local.

Fig. 8. Surface enzymatic test results.

de suspension lors de la récupération de la goutte avec la micropipette, par exemple). En revanche, lorsque la goutte a été en contact avec l'enzyme, le nombre de colonies bactériennes développées sur gélose et normalisé par rapport au témoin, diminue fortement : il est alors compris entre 0 à $10^{-2} \mathrm{cfu} / \mathrm{mL}$.

Ainsi, lorsque la suspension bactérienne est déposée sur un échantillon non fonctionnalisé pendant une nuit, la plupart des bactéries est récupérée. Cela signifie, d'une part, que les bactéries ne sont pas restées adsorbées sur la surface, et d'autre part, que le dépôt de bactéries sur la surface non fonctionnalisée n'altère pas les bactéries, puisque celles-ci sont ensuite capables de se développer normalement sur gélose. Quand la suspension bactérienne est mise en contact une nuit avec la surface fonctionnalisée par l'enzyme, très peu de bactéries viables et cultivables, présentes à l'origine dans la goutte sont récupérées. Deux hypothèses peuvent être avancées : soit les bactéries sont restées adsorbées sur la surface, soit elles ont été inactivées ou tuées par l'enzyme. Il a été vérifié précédemment que sur surface non fonctionnalisée quasiment toutes les bactéries étaient récupérées (Fig. 8), ce qui élimine a priori la première hypothèse. Ainsi, ce test permet d'attester l'efficacité des enzymes greffées, c'est-à-dire leur aptitude à empêcher l'adhésion significative des bactéries en contact avec la surface sur laquelle ces enzymes ont été immobilisées.
Un test complémentaire a permis de s'assurer que l'enzyme greffée ne désorbait pas dans la goutte de suspension bactérienne déposée (Fig. 9 et 10). Les conditions opératoires (milieux, température, ... ) sont identiques à celles du test enzymatique local.

Lorsqu'une goutte de tampon PBS est déposée sur la surface fonctionnalisée par l'enzyme, pendant une nuit, puis récupérée et mélangée avec une suspension bactérienne pendant une nuit également, aucune bactérie n'est éliminée (Fig. 10) : même nombre de bactéries que pour le témoin. Cela signifie qu'il n'y avait pas d'enzyme avec une activité enzymatique significative dans la goutte de PBS mélangée avec la suspension, et donc qu'aucune enzyme n'avait été préalablement libérée en quantité mesurable dans cette goutte de PBS. De plus, les conditions opératoires étant identiques à celles du test enzymatique, l'absence d'activité n'est pas due à une dénaturation de l'enzyme dans ces conditions particulières d'essai.

\section{Conclusion}

Ce travail a permis de modifier une surface d'acier inoxydable de manière à $y$ limiter l'adhésion d'une bactérie modèle. Plusieurs étapes ont été nécessaires au greffage sur la surface d'une enzyme capable de développer une activité biocide locale vis-àvis de la suspension bactérienne étudiée. Ces 




Fig. 9. Schéma du test de désorption.

Fig. 9. Desorption test drawing.

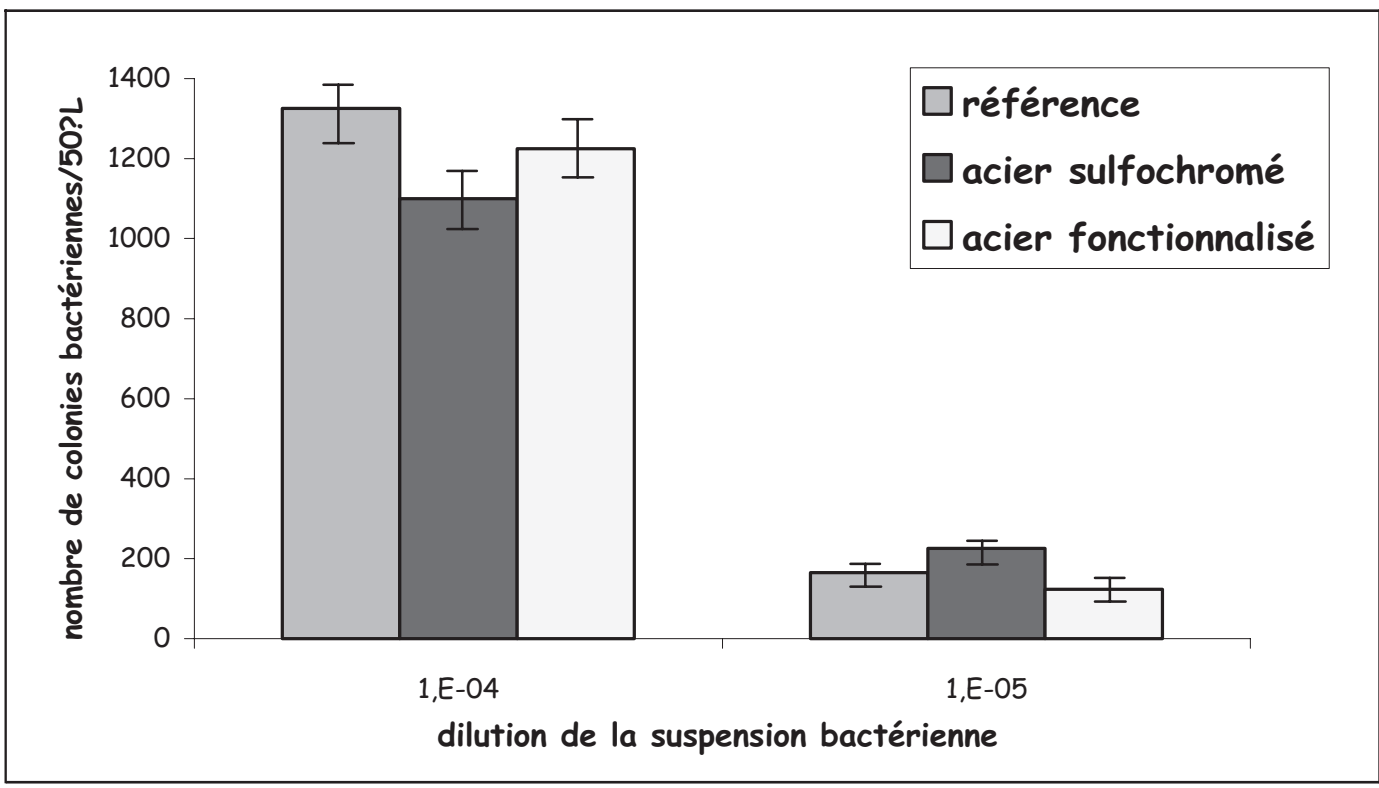

Fig. 10. Résultats du test de désorption.

Fig. 10. Desorption test results.

étapes successives ont été caractérisées à la fois par XPS et par PM-IRRAS : le couplage de ces deux techniques a permis de vérifier que les différentes molécules - PEI, GA et HEWL - étaient greffées de façon stable à la surface d'acier inoxydable (stabilité après plusieurs cycles de rinçage). Une fois la modification de la surface validée, l'efficacité de l'enzyme greffée a été testée vis-à-vis d'une suspension bactérienne modèle. Il a été vérifié que l'enzyme greffée avait une activité biocide locale capable d'empêcher l'adhésion des bactéries en contact avec la surface.

Cette modification de surface n'est pas toxique pour l'environnement puisque, d'une part, l'enzyme n'agit que localement, à la surface du matériau sur lequel elle est greffée, et d'autre part, elle n'est pas relarguée dans le milieu.

Le travail présenté concerne une enzyme modèle qui agit vis-à-vis d'une suspension bactérienne spécifique. Cependant, le mode de greffage exposé peut s'appliquer à tout type d'enzymes : les perspectives d'étude sont donc d'immobiliser des enzymes plus « universelles » telles que la subtilisine [28] et, éventuellement, plusieurs types d'enzymes simultanément, de manière à créer une surface efficace vis-à-vis d'une gamme plus large de bactéries. Alors, les surfaces 
pourront être testées en milieu réel, c'est-àdire en immergeant la surface dans un milieu bactérien représentatif du milieu marin.

\section{Références}

[1] C. Alzieu, Ocean Coast. Manage. 40 (1998) 2336

[2] I. Omae, Chem. Rev. 103 (2003) 3431-3448

[3] H.A. Videla, Inter. Biodeter. Biodegr. 49 (2002) 259-270

[4] E.Z. Ron, E. Rosenberg, Environ. Microbiol. 3 (2001) 229-236

[5] C. Hugues, C. Bressy, P. Bartolomeo, A. Margaillan, Eur. Polym. J. 39 (2003) 319-326

[6] C. Tamerler, S. Dincer, D. Heidel, M. Hadi Zareie, M. Sarikaya, Prog. Org. Coat. 47 (2003) 267-274

[7] N. Nath, J. Hyun, H. Ma, A. Chilkoti, Surf. Sci. 570 (2004) 98-110

[8] F. Zhang, E.T. Kang, K.G. Neoh, P. Wang, K.L. Tan, Biomaterials 22 (2001) 1541-1548

[9] R.F. Brady Jr., Prog. Org. Coat. 35 (1999) 31-35

[10] J.M.E. Harris, Poly(ethylene glycol) Chemistry - Biotechnical and Biomedical Applications, Plenum Press, New York, 1992

[11] M.E. Pettitt, S.L. Henry, M.E. Callow, J.A. Callow, A.S. Clare, Biofouling 20 (2004) 299311

[12] K. Turner, M. Serantoni, A. Boyce, G. Walsh, Process Biochem. 40 (2005) 3377-3382

[13] S.J. Novick, J.S. Dordick, Biomaterials 23 (2002) 441-448
[14] J.H. Lee, H.B. Lee, J.D. Andrade, Prog. Polym. Sci. 20 (1995) 1043-1079

[15] S.-W. Lee, P.E. Laibinis, Biomaterials 19 (1998) 1669-1675

[16] S.I. Jeon, J.H. Lee, J.D. Andrade, P.G. De Gennes, J. Colloid Interf. Sci. 142 (1991) 149158

[17] K.L. Prime, G.M. Whitesides, J. Amer. Chem. Soc. 115 (1993) 10714-10721

[18] R.L.C. Wang, H.J. Kreuzer, M. Grunze, J. Phys. Chem. B 101 (1997) 9767-9773

[19] A. Halperin, Langmuir 15 (1999) 2525-2533

[20] S.R. Sheth, D. Leckband, Proc. Natl. Acad. Sci. USA 94 (1997) 8399-8404

[21] P. Kingshott, J. Wei, D. Bagge-Ravn, N. Gadegaard, L. Gram, Langmuir 19 (2003) 6912-6921

[22] P. Paula, M.F. Heidi, J. Adhes. Sci. Technol. 15 (2001) 869-888

[23] D.A. Puleo, Biomaterials 17 (1996) 217-222

[24] J. Wei, D.B. Ravn, L. Gram, P. Kingshott, Colloid. Surface. B 32 (2003) 275-291

[25] P. Appendini, J.H. Hotchkiss, Packaging Technol. Sci. 10 (1997) 271-279

[26] J.H. Scofield, J. Electron Spectrosc. 8 (1976) 129-137

[27] P.E. Laibinis, C.D. Bain, G.M. Whitesides, J. Phys. Chem. 95 (1991) 7017-7021

[28] C. Rubio, Compréhension des mécanismes d'adhésion des biofilms en milieu marin en vue de la conception de nouveaux moyens de prévention, Thèse, Université de Paris 6 , 2002 